\title{
Prostate Stem Cell Antigen Is an Endogenous lynx1-Like Prototoxin That Antagonizes $\alpha 7$-Containing Nicotinic Receptors and Prevents Programmed Cell Death of Parasympathetic Neurons
}

\author{
Martin Hruska, ${ }^{1}$ Julie Keefe, ${ }^{1}$ David Wert, ${ }^{1}$ Ayse Begum Tekinay, ${ }^{2}$ Jonathan J. Hulce, ${ }^{1}$ Ines Ibañez-Tallon, ${ }^{3}$ \\ and Rae Nishi ${ }^{1}$ \\ ${ }^{1}$ Department of Anatomy and Neurobiology, University of Vermont College of Medicine, Burlington, Vermont 05405, ${ }^{2}$ Laboratory of Molecular Biology, \\ Howard Hughes Medical Institute, Rockefeller University, New York, New York 10021, and ${ }^{3}$ Max-Delbrück-Center, 13125 Berlin, Germany
}

Vertebrate $\alpha$-bungarotoxin-like molecules of the Ly- 6 superfamily have been implicated as balancers of activity and survival in the adult nervous system. To determine whether a member of this family could be involved in the development of the avian ciliary ganglion, we identified 6 Gallus genes by their homology in structure to mouse lynx1 and lynx2. One of these genes, an ortholog of prostate stem cell antigen (psca), is barely detectable at embryonic day (E) 8, before neuronal cell loss in the ciliary ganglion, but increases $>100$-fold as the number of neurons begins to decline between E9 and E14. PSCA is highly expressed in chicken and mouse telencephalon and peripheral ganglia and correlates with expression of $\alpha 7$-containing nicotinic acetylcholine receptors ( $\alpha 7$-nAChRs). Misexpressing PSCA before cell death in the ciliary ganglion blocks $\alpha 7-\mathrm{nAChR}$ activation by nicotine and rescues the choroid subpopulation from dying. Thus, PSCA, a molecule previously identified as a marker of prostate cancer, is a member of the Ly- 6 neurotoxin-like family in the nervous system, and is likely to play a role as a modulator of $\alpha 7$ signaling-induced cell death during development.

\section{Introduction}

Nicotinic signaling has been implicated in controlling programmed cell death during the development of the nervous system. By far the most effective means of rescuing spinal cord motor neurons from dying is to treat embryos with nicotinic antagonists that block neuromuscular transmission such as D-tubocurarine (dTC) or $\alpha$-bungarotoxin ( $\alpha$ btx) (Pittman and Oppenheim, 1979). In contrast, in the avian ciliary ganglion, up to $90 \%$ of the neurons in the avian ciliary ganglion are rescued by antagonists of $\alpha 7$-containing nicotinic acetylcholine receptors (nAChRs) such as $\alpha$ btx and $\alpha$-methyllycaconitine (MLA) (Bunker and Nishi, 2002; Hruska et al., 2007); however, nonselective $\mathrm{nAChR}$ antagonists that completely block ganglionic transmission exacerbate cell death (Wright, 1981; Meriney et al., 1987; Maderdrut et al., 1988). In cell culture, nicotine promotes survival of ciliary ganglion neurons, but it does so in the absence of

\footnotetext{
Received May 13, 2009; revised 0ct. 1, 2009; accepted 0ct. 9, 2009.

This project was funded by Grant DA17784 from the National Institutes of Health to R.N. The real-time PCR and calcium imaging were made possible by the Neuroscience Centers of Biomedical Research Excellence (Grant 5 P20 RR016435). We are grateful to Dr. Nathanial Heintz at Rockefeller University for providing us with anti-mouse lynx 1 antibodies that started this project and for his insights. We also thank Priscilla Kimberly and Sondra Sammut for excellent technical support.

A. B. Tekinay's present address: UNAM, Institute of Materials Science and Nanotechnology, Bilkent University, 06800 Ankara, Turkey.

Correspondence should be addressed to Rae Nishi, Department of Anatomy and Neurobiology, HSRF 406, University of Vermont College of Medicine, 149 Beaumont Avenue, Burlington, VT 05405. E-mail: rnishi@uvm.edu.

DOI:10.1523/JNEUROSCI.2271-09.2009

Copyright $\odot 2009$ Society for Neuroscience $\quad$ 0270-6474/09/2914847-08\$15.00/0
}

trophic support (Pugh and Margiotta, 2000). Thus, the contribution of nicotinic signaling to neuronal survival during development is complex.

Endogenous prototoxins that are homologous to snake venom neurotoxins modulate signaling through nAChRs. These molecules are members of the Ly6/neurotoxin (lynx) family characterized by their cysteine-rich motif that predicts their folding into the typical three-fingered loop structure of $\alpha$-bungarotoxin (Miwa et al., 1999; Chimienti et al., 2003). Members of this family that are GPI-linked and expressed in the nervous system are lynx1, lynx2, Ly6H (Horie et al., 1998; Miwa et al., 1999; Dessaud et al., 2006); others are secreted and expressed in non-neuronal cells, e.g., SLURP-1 and SLURP-2 (Chimienti et al., 2003; Tsuji et al., 2003). Mouse lynx1 binds to and alters the kinetics of nAChRs (Ibañez-Tallon et al., 2002). Furthermore, cortical neurons of mice lacking lynx1 have an enhanced sensitivity to nicotine that potentiates excitotoxicity, whereas aging lynx1-null mice exhibit exacerbated neurodegeneration in the brain that is enhanced by nicotine and attenuated by loss of nAChRs (Miwa et al., 2006).

In the present study we determined whether the expression of endogenous lynx-like molecules could play a role during the development of the avian ciliary ganglion. The ganglion contains two types of neurons, ciliary and choroid, and both receive cholinergic innervation that is detectable at embryonic day (E) 5.5 and complete by E8 (Landmesser and Pilar, 1972). Over half of the ciliary and choroid neurons are lost between E8 and E14 (Landmesser and Pilar, 1974). By E8, all neurons express homo- 
meric $\alpha 7$-nAChRs and heteromeric $\alpha 3^{\star}$ nAChRs containing $\alpha 3$, $\alpha 5, \beta 4$ subunits (Blumenthal et al., 1999; McNerney et al., 2000) and sometimes $\beta 2$ (Conroy and Berg, 1995). Using mouse lynx1 and lynx2 we screened for homologous molecules in the chicken and identified 6 genes. Of these, one is induced in the ciliary ganglion during the period of cell loss and is the chicken ortholog of human prostate stem cell antigen ( $p s c a)$. PSCA is developmentally regulated and its expression reduces $\alpha 7$ nAChR-mediated increases in intracellular $\mathrm{Ca}^{2+}$ and promotes survival of choroid neurons. We conclude that PSCA, a molecule previously identified as upregulated in prostate cancer, is also a lynx-like prototoxin molecule that functions in the developing nervous system.

\section{Materials and Methods}

Identification of chicken lynx sequences. Mouse lynx1 and lynx2 protein sequences were blasted against chicken TIGR gene indices (http://www. tigr.org/tdb/tgi/) and we chose 6 sequences that fit the following criteria: (1) having the Ly6 domain as the only functional domain; (2) matching the exon-intron structures of lynxl and other prototoxins that have the Ly6 domain. The accession numbers of the sequences that we discovered are given in supplemental Table 1, available at www.jneurosci.org as supplemental material. Based on predicted sequences, sequence-specific primers were used to identify the expression profile of above lynx related transcripts in the ciliary ganglion at E8 and E15 (for primer sequences, see supplemental Table 1, available at www.jneurosci.org as supplemental material).

RNA extraction and PSCA cloning. Tissues were isolated at the indicated stages of development and rapidly frozen on dry ice. Total RNA was extracted by using TRI Reagent (Molecular Research Center). The cDNA was reverse-transcribed from $2 \mu \mathrm{g}$ of total RNA by using oligo-dT and Superscript II reverse transcriptase (Invitrogen). The full-length PSCA was obtained by PCR using forward primer (5' CCATGGTAATGAAGGTTTTCTTCATCCTCC $3^{\prime}$ ) that attached an Ncol restriction site to the $5^{\prime}$ end of a PSCA sequence and reverse primer (5' GGATCCCTTCACAGTCTGTTGTTCAGG $3^{\prime}$ ) that added a BamH1 restriction site to the 3 ' end of the PSCA sequence. The 369 base pair PCR product was cloned into Ncol and BamH1 cloning sites on pSlax13Nco vector and sequencing confirmed that it was the accurate, full-length chicken PSCA sequence.

Real-time quantitative PCR. Tissues were isolated at the indicated ages and rapidly frozen on dry ice. Total RNA was extracted and cDNA was synthesized as described above. The expression profiles of $\alpha 7$ and PSCA were assessed on the 7500 Fast Real-Time PCR System (Applied Biosystems) using TaqMan probes and primers designed using a website at Whitehead Institute at MIT http://frodo.wi.mit.edu/primer3/ (see supplemental Table 2, available at www.jneurosci.org as supplemental material). Probes were labeled with 6 FAM reporter dye at their $5^{\prime}$ ends and black hole quencher (BHQ) at their $3^{\prime}$ ends. The constitutive gene control used to normalize gene expression was chicken $\mathrm{S} 17$ ribosomal binding protein (Chrps) (Darland et al., 1995). Relative transcript expression and number was determined using Sequence Detection Software (SDS) version 1.4. PSCA expression in mouse tissues was quantified and normalized to $\beta$-actin using Assays on Demand from Applied Biosystems (PSCA: Mm00452908_m1; $\beta$-actin: Mm00607939_s1).

Expression of PSCA with a viral vector. The PSCA sequence was cloned into the Slax13NCO1 shuttle vector using 5' $\mathrm{Ncol}$ and $3^{\prime} \mathrm{BamH} 1$ sites (Morgan and Fekete, 1996); the insert was removed from Slax13NCO1 by cutting with Cla1, and cloned into the avian retroviral vector RCASBP(A) (Federspiel and Hughes, 1997). Infective RCASBP(A)PSCA viral particles were generated by transfecting DF-1 chicken fibroblast cells with $800 \mathrm{ng}$ of RCASBP(A)-PSCA plasmid using Mirus TransIT-LT transfection reagent. Conditioned media containing viral stocks collected from DF-1 cells were concentrated $\sim 20$-fold by ultracentrifugation at $90,000 \times g$ at $4^{\circ} \mathrm{C}$ for $3 \mathrm{~h}$ (Morgan and Fekete, 1996). Concentrated stocks were titered by limiting dilution and infectivity of cells as measured by staining with p27gag antibody. Stocks with concen- tration of $>10^{8}$ infectious particles/ml were used for in vivo injection. Viral particles $(60-120 \mathrm{nl})$ were injected into the mesencephalic enlargement of the neural tube of Hamburger/Hamilton stage (St.) $8-9$ or St 10-13 embryos using a Drummond Nanoject microinjector. The shells were sealed with a glass coverslip and sterile vacuum grease and incubated at $37^{\circ} \mathrm{C}$ to the desired stage.

Calcium imaging. Acutely isolated ciliary ganglion neurons were loaded with Fura-2 AM (Invitrogen) dissolved in DMSO at final concentration of $5 \mu \mathrm{M}$ with $2 \%$ Pluronic F-127 (Invitrogen). Neurons were loaded at room temperature for $30 \mathrm{~min}$ in the dark. Calcium signals were recorded by exposure to alternating wavelength ( 340 and $380 \mathrm{~nm}, 50 \mathrm{~ms}$ ) generated by a Xenon light source and Lambda DG-4 ultra high-speed wavelengths switcher (Sutter Instruments). Fluorescent responses were recorded using an Orca-ER digital camera (Hamamatsu). Paired 340/380 ratio images were acquired at $4 \mathrm{~s}$ intervals with Metafluor 5.0r5 software (Molecular Devices). Drugs were dissolved in chicken physiological buffer (containing, in mM: $145 \mathrm{NaCl}, 5.4 \mathrm{KCl}, 0.8 \mathrm{MgCl}_{2}, 5.4 \mathrm{CaCl}_{2}, 5$ glucose, 13 HEPES, pH 7.4). Voltage-gated sodium and calcium channels were blocked when nicotine was applied by supplementing the perfusion medium with $600 \mathrm{~nm}$ tetrodotoxin (Tocris Bioscience) and $200 \mu \mathrm{M}$ cobalt chloride (Sigma-Aldrich), respectively. Nicotine (Sigma-Aldrich) (10 $\mu \mathrm{M})$ was applied for $20 \mathrm{~s}$ to activate nAChRs. $\alpha 7$-nAChRs were inhibited by perfusing the neurons with $50 \mathrm{~nm} \alpha$-methyllycaconitine citrate hydrate (MLA; Sigma-Aldrich) for $1 \mathrm{~min}$ or preincubating with $50 \mathrm{~nm} \alpha$ btx (Sigma-Aldrich) for $30 \mathrm{~min}$ at $25^{\circ} \mathrm{C}$. On completion of these experiments, the extent of dye loading was determined by activating voltagegated calcium channels with high potassium perfusion solution $(25 \mathrm{~mm}$ $\mathrm{KCl}$ ) in the absence of TTX or cobalt chloride. After the initial recordings were performed, background was subtracted from every image acquired and new ratios were calculated using the Metafluor $5.0 \mathrm{r} 5 \mathrm{soft}-$ ware. The ratios were then exported into the Microsoft Excel spreadsheet for calculations.

Tissues. Tissues were prepared for immunohistochemistry and in situ hybridization as follows. Ciliary ganglia and brainstems from E14 embryos were harvested, fixed in Zamboni's fixative for $48 \mathrm{~h}$ at $4^{\circ} \mathrm{C}$, washed, then equilibrated in $30 \%$ sucrose at $4^{\circ} \mathrm{C}$. Tissue was embedded in Microm cryoembedding compound (Richard Allen Scientific), sectioned on a Microm HM 560 cryostat (Richard Allen Scientific) at $30 \mu \mathrm{m}$ (for immunohistochemistry) and at $20 \mu \mathrm{m}$ for in situ hybridization and collected on Superfrost Plus slides (Fisher Scientific). Sections were postfixed in Zamboni's vapors for $15 \mathrm{~min}$ at $37^{\circ} \mathrm{C}$, submerged in Zamboni's fixative for additional $15 \mathrm{~min}$ at $25^{\circ} \mathrm{C}$, washed in PBS and blocked. Primary antibodies were incubated overnight at $4^{\circ} \mathrm{C}$ and secondary antibodies were incubated $2 \mathrm{~h}$ at room temperature.

In situ hybridization. In situ hybridization was performed as described by the D. Anderson laboratory at Caltech (http://wmc.rodentia.com/docs/Big In_Situ.html) with the following modifications: sections collected on slides were treated with $100 \mu \mathrm{g} / \mathrm{ml}$ proteinase K (Sigma-Aldrich) for $10 \mathrm{~min}$ at room temperature; hybridization was performed with digoxigenin-labeled UTP (Roche)-labeled sense or antisense riboprobes synthesized using an RNA transcription kit (Ambion) from a full-length Gallus clone gcag0014c.j.08 corresponding to the cDNA we cloned, which was obtained from the French National Institute for Agricultural Research Animal Genomics Collection (http://www.international.inra.fr); and riboprobes were subjected to alkaline hydrolysis until average transcript sizes were 300-400 bp.

Primary antibodies. Mouse monoclonal 39.4D5 [Developmental Studies Hybridoma Bank (DSHB), University of Iowa, Iowa City, IA], which recognizes Islet- 1 and Islet-2, transcription factors expressed in all ciliary ganglion neurons throughout development (Lee et al., 2001) at 1:100 dilution of the culture supernatant prepared in the Nishi Laboratory from hybridomas obtained from DSHB; mouse anti-Hu C/D (Invitrogen), which recognizes a neuron-specific RNA binding protein (Marusich and Weston, 1992; Lee et al., 2001) at 1:250 dilution of the culture supernatant; rabbit anti-p27gag (Charles River SPAFAS), which recognizes avian sarcoma gag p27 (Wang et al., 1976) at 1:1000; rat anti-somatostatin (product number YMC1020, Accurate Chemical) diluted 1:100. Sheep anti-digoxigenin (Roche Applied Science) was used at 1:500 and visualized with goat antisheep coupled to alkaline phosphatase (1:1000; Roche). 
A

Mouse Lynx Mature
Ch1Ly Mature
Ch2Ly Mature
Ch3Ly Mature
Ch4Ly Mature
Ch5Ly Mature
Ch6Ly Mature

Mouse Lynx Mature
Ch1Ly Mature
Ch2Ly Mature
Ch3Ly Mature
Ch4Ly Mature
Ch5Ly Mature
Ch6Ly Mature

Mouse Lynx Mature Ch1Ly Mature Ch2Ly Mature Ch3Ly Mature Ch4Ly Mature Ch5Ly Mature Ch6Ly Mature

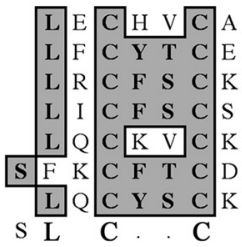

10

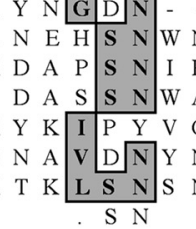

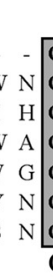

20

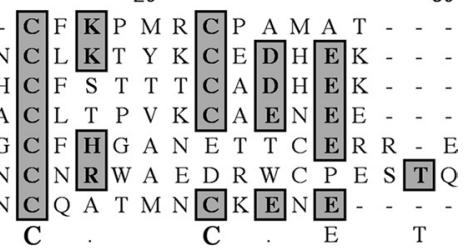

50

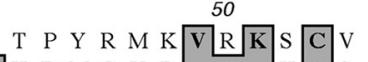

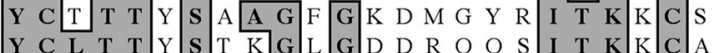

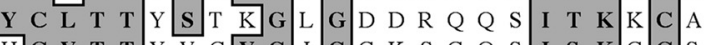

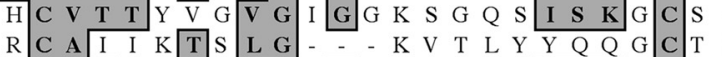

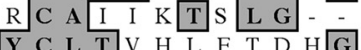

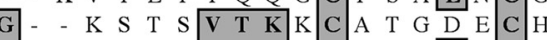

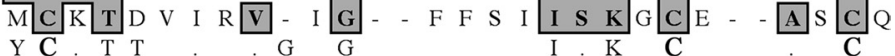
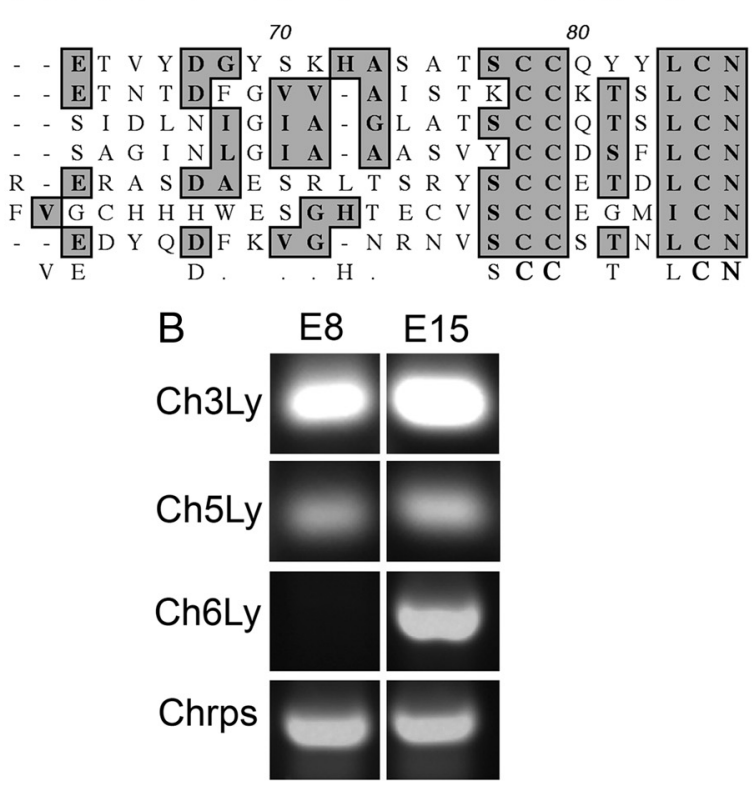

Figure 1. Molecules with homology to the Ly6/Neurotoxin-like superfamily in chicken ciliary ganglion. A, Amino acid sequences of 6 mature chicken proteins identified by their homology to mouse lynx 1 and $/ y n x 2$ are shown aligned to mouse lynx 1 . All sequences contain the Ly 6 functional domain (in bold at the bottom). Boxed residues are amino acids that are identical to those in mouse lynx1. B, Sequence-specific primers to each of the 6 chicken sequences were used to amplify cDNA from E8 and E14 ciliary ganglia. Three sequences are expressed in the ciliary ganglion. The ch3Ly and ch $5 L y$ are present at E8 and E14; however, ch6Ly is only expressed at E14. Chrps is a constitutive gene control.

Secondary antibodies. Biotinylated anti-mouse (Vector Laboratories) at 1:250; biotinylated anti-rabbit (Vector Laboratories) at 1:250; goat anti-mouse Cy3 (Jackson ImmunoResearch) at 1:750; goat anti-rabbit Alexa 488 (Invitrogen) at 1:750; and goat anti-rat Cy3 (Jackson ImmunoResearch) at 1:750. Images of ciliary ganglia and midbrain were acquired with $20 \times$ objective using Nikon C1 confocal scanner (Nikon Instruments) attached to a Nikon Eclipse E800 microscope (Micro Video Instruments).

Design-based stereology. Serially sectioned ciliary ganglia (cut at 30 $\mu \mathrm{m}$ ) were prepared for designed-based stereology as previously described (Lee et al., 2001; Bunker and Nishi, 2002). Islet-1/2-positive nuclei (representing all neurons) together with somatostatin-positive cell bodies (representing all choroid neurons) were counted using the Optical Fractionator Probe of Stereo Investigator (MBF Bioscience) in conjunction with a Nikon Optiphot 2 microscope with a Hitachi HVC20 camera, Heidenhahn focus encoder, and a motorized, computer-driven $\mathrm{X}-\mathrm{Z}$ stage (all microscope attachments provided by MBF Biosciences). To avoid inaccuracies caused by cutting artifacts and double counting between adjacent sections, an upper guard of $4 \mu \mathrm{m}$ and lower guard of 7 $\mu \mathrm{m}$ were used (Bunker and Nishi, 2002). Spacing between sampling sites (grid size) was set such that 13-15 sampling sites were counted per section, which yielded 100-300 objects per each ciliary ganglion. The

90

number of ciliary neurons was calculated by subtracting the number of somatostatin-positive neurons from the total number of neurons per ciliary ganglion (Bunker and Nishi, 2002).

\section{Results}

\section{Identification of chicken}

lynx-like sequences

Since mouse lynx1 interacts with $\alpha 7$ nAChRs from chicken (Ibañez-Tallon et al., 2002), we used the amino acid sequences of mouse lynx1 and lynx2 to search a chicken expressed sequence tag (EST) database for proteins that had the Ly6 domain as the only functional domain and whose genes had similar exonintron structures to those of lynxl and other toxins that have the Ly6 domain. Six sequences that matched these criteria were identified (Fig. 1A). All 6 sequences are cysteine-rich and 10 of the cysteine residues are aligned across all molecules. The sequences also encode an $\mathrm{N}$-terminal signal sequence and a C-terminal consensus sequence for addition of a glycosylphosphatidylinositide linkage to the membrane. When analyzed by SMART (Simple Modular Architecture Research Tool; http://smart.embl-heidelberg.de/), all molecules are also predicted to fold into a three-fingered coiled structure similar to that of mouse lynx1 and the cobra venom neurotoxins (Gumley et al., 1995). This striking structural homology suggests that these chicken molecules can interact with nicotinic acetylcholine receptors as shown for mouse lynx1 (IbañezTallon et al., 2002).

To determine whether any of the 6 EST transcripts were expressed in a developmentally regulated pattern in the ciliary ganglion, we used sequence-specific primers (supplemental Table 1, available at www. jneurosci.org as supplemental material) to amplify cDNA from ciliary ganglia collected at E8, while cell death is occurring, and E14, at which time cell death has ceased (Lee et al., 2001). Three sequences, ch3Ly, ch5Ly and ch6Ly were expressed in E14 ganglia; however, ch3Ly and ch5Ly were also expressed at E8 (Fig. $1 \mathrm{~B}$ ). In contrast, no expression of ch6Ly is detectable at E8 (Fig. $1 B$ ). Identity of the amplified gene product as ch6Ly was confirmed by sequencing. The remaining transcripts are not expressed in the embryonic ciliary ganglion (data not shown). Ch6Ly encodes a pro-protein of 122 aa, which corresponds to a molecular weight of 11,160 Da. The mature GPIlinked protein is $8051 \mathrm{Da}$. When ch6Ly is used to search Entrez, it matches mouse prostate stem cell antigen ( $p s c a)$, with which it shares $40 \%$ amino acid identity and $80 \%$ homology (Fig. 2A). Like ch6Ly, the open reading frame of mouse psca is similar to other Ly6 superfamily members in the alignment of cysteine groups and contains the Ly6 domain. In addition, the genomic structure of mouse psca is identical to that of ch6Ly, mouse lynxl, mouse $l y 6 h$, and $\alpha$-bungarotoxin, with conserved intron/exon breaks (Fig. 2 B). Thus, ch6Ly is likely to be the chicken ortholog 
of prostate stem cell antigen ( $p s c a)$, which is a Ly6 family member whose expression becomes upregulated in prostate tumors (Reiter et al., 1998).

\section{Expression pattern of chicken PSCA}

To determine the specificity of chicken PSCA expression, we quantified transcripts in a variety of tissues in E14 chicken embryos using real-time PCR and normalized these levels to chicken ribosomal binding protein s17 (CHRPS), a constitutively expressed housekeeping gene that is abundant in all tissues. Expression of PSCA in selected tissues was determined relative to that of heart, which contained barely detectable levels (Fig. $3 A$ ). Levels of PSCA transcripts in pectoral muscle, liver, ovary and testes (data not shown) are comparable to that of heart. In the nervous system, the cerebellum also has very low levels; however, the telencephalon has fivefold greater levels of PSCA mRNA than cerebellum. The highest levels of PSCA occur in autonomic ganglia: the ciliary ganglion, which is parasympathetic, contains $>20$-times that of heart, and paravertebral lumbar sympathetic ganglia contain 10-times more PSCA. Dorsal root ganglia also express higher levels of PSCA mRNA than heart, but considerably less than sympathetic or ciliary ganglia.

Since our observation that PSCA is enriched in telencephalon was contrary to the original report that PSCA could not be detected in northern blots of brain mRNA (Reiter et al., 1998), we determined whether adult mouse tissues exhibit an expression pattern of PSCA mRNA similar to that of chicken embryos. We isolated neural and non-neural tissues and quantified relative abundance of mouse PSCA normalized to $\beta$-actin using real-time PCR, which is more sensitive than the northern blot approach previously used. As in late stage chicken embryos, the nervous tissues isolated from adult mice contain significantly higher levels of PSCA than non-neural tissues, with the highest levels found in the superior cervical ganglion, which is sympathetic (Fig. $3 B$ ). In fact, all the tissues that exhibit high levels of PSCA in the chicken embryo also express high levels of $\alpha 7$-nAChRs (Fig. $3 C$ ). Thus, PSCA is expressed at very high levels in neural tissues both in mammals and avian species, and its level of expression correlates with the expression of the $\alpha 7$-nAChR subunit (Fig. $3 C, D)$, suggesting a relationship between PSCA and $\alpha 7$ signaling.

In ciliary ganglia, which express some of the highest levels of $\alpha 7 \mathrm{nAChRs}$ per

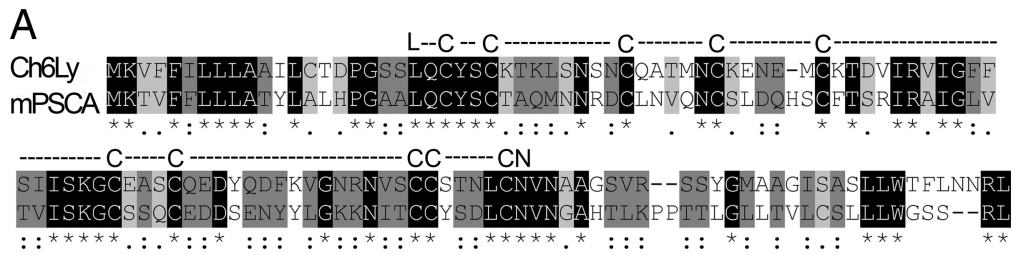

B

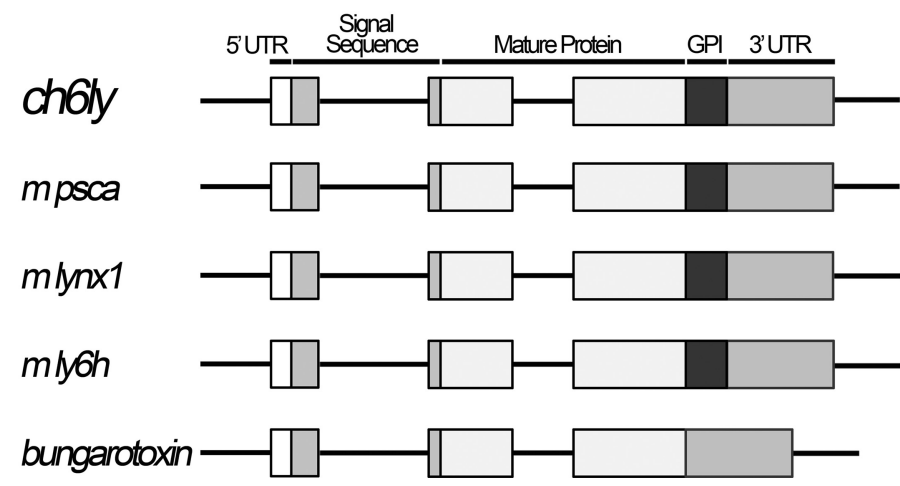

Figure 2. Ch6Ly is psca.A, The amino acid sequence of ch6Ly was used to search NCBl and Ensembl databases and found to have the most significant match to mouse psca. The two sequences share $80 \%$ homology and $40 \%$ identity. Black highlight indicates positions with single, fully conserved residues. Dark gray highlight indicates that the strong amino acid groups are fully conserved. Light gray highlight represents the fully conserved weak amino acid groups. Both molecules contain the cysteine-rich Ly 6 domain (shown above the amino acid sequence) with conserved N-terminal leucine and C-terminal asparagine. B, Representation of the open reading frame of the genes encoding ch6Ly and mouse psca compared with the other members of Ly6/Neurotoxin superfamily such as lynx 1, ly6 $h, \alpha$-bungarotoxin. The ch6ly and psca have the same intron/exon breaks as the other members of the superfamily. The 5' UTR sequence, signal sequence, mature protein containing Ly6 domain, hydrophobic GPI anchor and 3'UTR sequence are also conserved between ch6ly, psca and other members of the family.
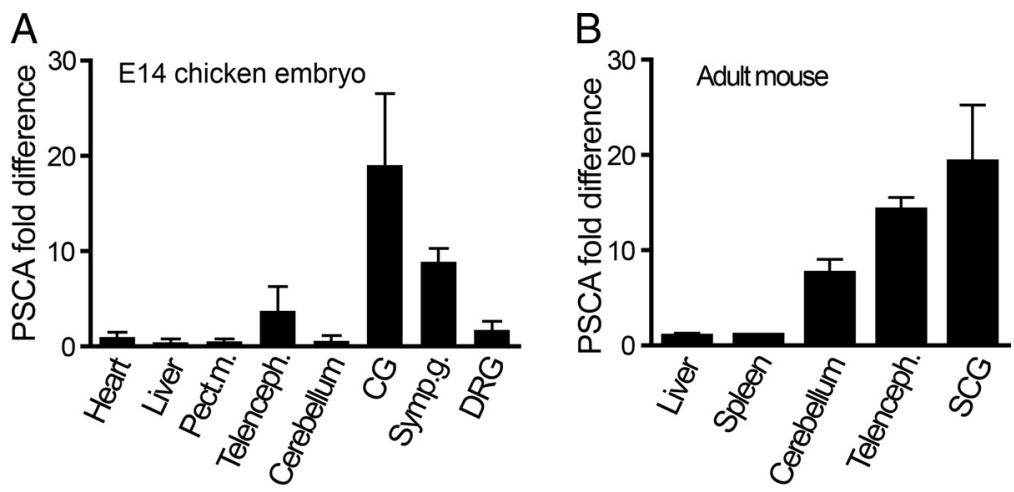

C

D
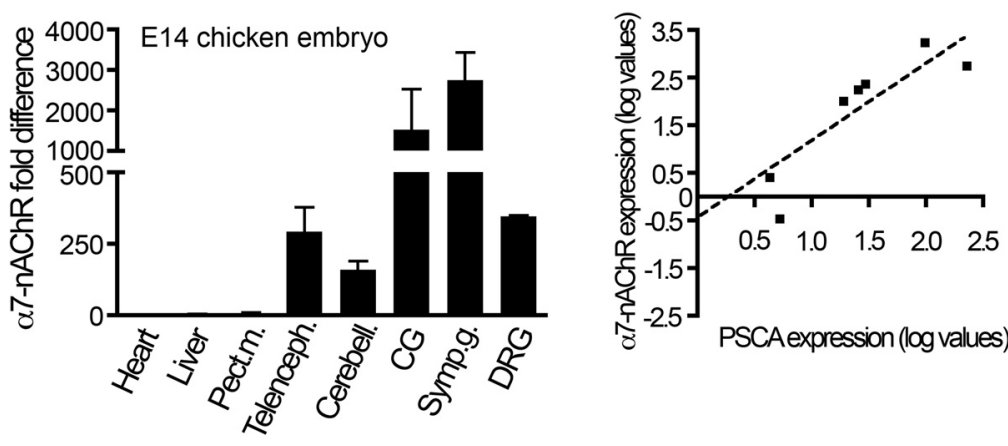

Figure 3. PSCA transcripts are highly expressed in nervous tissues and correlate with $\alpha 7 \mathrm{nAChR}$ expression. $A$, A variety of neural and non-neural tissues were dissected from E14 chicken embryos and mRNA encoding chicken PSCA were quantified using quantitative realtime PCR and normalized to Chrps. PSCA expression was highly expressed in neural tissues, particularly in peripheral autonomic ganglia (CG: ciliary ganglia; symp. g: sympathetic ganglia). PSCA is 20 times more abundant in ciliary ganglion than in non-neural tissues. $\boldsymbol{B}$, Similar pattern of PSCA expression is observed in tissues isolated from adult mouse. Neural tissues and peripheral ganglia contain 10-20-fold higher levels of PSCA than non-neural tissues. C, $\alpha 7$ subunit of nAChR expression correlates with PSCA. By far, the highest levels of $\alpha 7$ expression are in ciliary and sympathetic ganglia. $\boldsymbol{D}$, The levels of $\alpha 7 \mathrm{nAChR}$ increase as PSCA expression increases, with a correlation coefficient of 0.8 . Values represent mean \pm SEM from at least 3 separate experiments. 


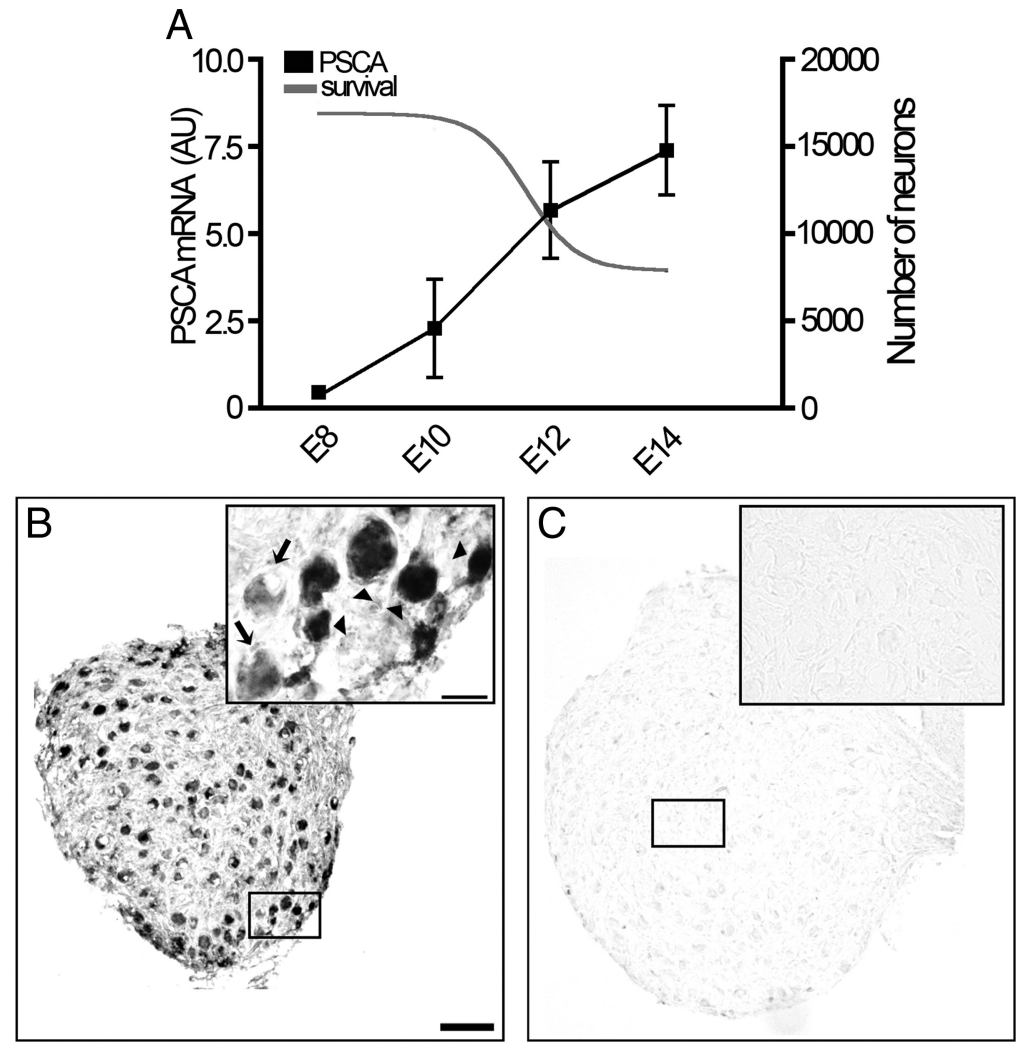

Figure 4. PSCA is expressed in neurons of the ciliary ganglion. $A$, Between E8 and E14, as the number of ciliary ganglion neurons is reduced by 50\% [gray curve based on data from our previous work (Lee et al., 2001)], PSCA expression increases 15 -fold (black line). $\boldsymbol{B}$, In situ hybridization was performed on sections from E15 ciliary ganglia using digoxigenin-labeled antisense riboprobe for chicken PSCA. Staining seems particularly intense in some small neurons on the periphery of the ganglion that may be choroid neurons (arrowheads, inset); however other larger neurons are also stained. PSCA staining is less intense in other neurons (arrows, inset). C, Sense probe showing only background staining. AU, Arbitrary units. Calibration bar $=100 \mu \mathrm{m}$, inset $=20 \mu \mathrm{m}$.

Table 1. Chronic application of $\alpha$ btx reduces upregulation of PSCA in the developing ciliary ganglion

\begin{tabular}{lllr}
\hline Target gene & Saline & $\alpha$-Bungarotoxin & Change \\
\hline PSCA & $171 \pm 17.7$ & $107 \pm 8.2^{*}$ & $63 \%$ \\
$\alpha 7$ & $184 \pm 41.7$ & $362 \pm 10.4^{*}$ & $200 \%$ \\
\hline
\end{tabular}

Chicken embryos were windowed at E3; then, $50 \mu \mathrm{g}$ per day of $\alpha$ btx dissolved in sterile saline or saline alone was applied in $50 \mu \mathrm{l}$ aliquots daily from E7 to E10. Ganglia were harvested at E11 and total RNA was extracted and reverse transcribed. Target gene expression was quantified in triplicate with quantitative real-time PCR using TaqMan probes. Values given are the mean \pm SE of RNA isolated from three independent groups of experiments, pooling 14-16 ganglia from each treatment. ${ }^{*} p<0.01$, one-way ANOVA with Tukey's post hoc test.

neuron, $\alpha 7$-nAChRs have previously been implicated in inducing cell death (Bunker and Nishi, 2002; Hruska et al., 2007). If PSCA attenuates cell death by modulating activation of $\alpha 7$ subunitcontaining nAChRs, then its expression should be low when cell death commences, and it should be upregulated as neurons in the ganglion extend axons to the periphery and initiate synaptogenesis. Accordingly, we used real-time PCR to quantify PSCA transcripts in ciliary ganglia isolated from E8 to E14 (Fig. 4A), which corresponds to a period when half the neurons are lost due to cell death. PSCA transcripts in ciliary ganglia are barely detectable at E8. However, midway through the cell loss period at E10-12, PSCA levels increase tenfold. This increase can be attenuated by chronic application of $\alpha$ btx at a concentration $(20 \mu \mathrm{g} / \mathrm{d})$ known to rescue neurons while the $\alpha 7 \mathrm{nAChR}$ transcript levels double, as might be expected if fewer neurons were dying (Table 1) (Bunker and Nishi, 2002). By E14, PSCA expression reaches highest levels, when it is 15 times more abundant than at E8 (Fig. 4A). After E14, which marks the end of cell loss in the ciliary ganglion, there is no further increase in PSCA transcript levels (data not shown). At E14, PSCA transcripts are detected in many neurons, but the relative levels of expression vary from low (Fig. $4 B$, arrows) to very high (Fig. $4 B$, arrowheads). Little or no signal is observed with the sense probe (Fig. 4C). Thus, PSCA mRNA correlates with the period of cell loss in the ciliary ganglion and is found in neurons, consistent with a possible role in modulating nicotinic signaling.

\section{PSCA blocks activation of $\alpha 7-$ nAChRs and rescues neurons from cell death}

To test whether PSCA could modify nicotine-induced responses in ciliary ganglion neurons, we used the retroviral vector RCASBP(A) to express PSCA at E8, an age at which PSCA mRNA is almost undetectable. We removed PSCA-expressing ciliary ganglia and vector-only infected controls at E8, plated the neurons on coverslips, and loaded them with the calcium-sensitive dye, Fura-2, to quantify intracellular calcium in response to rapidly perfused nicotine. To isolate nicotinic $\mathrm{Ca}^{2+}$ responses from $\mathrm{Ca}^{2+}$ influx due to the opening of voltage-gated ion channels, tetrodotoxin (TTX) and cobalt were added to the perfusion solution (see Materials and Methods).

In controls, nicotine induces a large increase in intracellular calcium, which is partially blocked by the $\alpha 7$ subunitspecific nicotinic cholinergic antagonist, MLA (Fig. 5A). The remaining response is blocked by dihydro $\beta$-erythroidin, an antagonist of $\alpha 3^{*}$ nAChRs (data not shown). When neurons infected with RCASBP(A)-PSCA are compared with those infected with open-RCASBP(A), there is no difference in the calcium signal induced by depolarization with $25 \mathrm{mM} \mathrm{KCl}$ in the absence of TTX and $\mathrm{Co}^{2+}($ Open $=0.52+0.04$ SEM; $n=30$; PSCA $=0.59+$ 0.06 SEM, $n=26$ ), indicating equal loading of Fura- 2 and good health of PSCA infected neurons. In neurons overexpressing PSCA, the mean nicotine-induced calcium response is reduced by $43 \%$ when compared with open vector-infected cells (Fig. $5 B$; $p<0.001$, one-way ANOVA, Bonferroni post test; open: $0.3 \pm$ 0.02 SEM, $n=69$; PSCA: $0.17 \pm 0.01$ SEM, $n=86$ ). This contrasts with the $69 \%$ reduction observed when neurons are infected with a tethered $\alpha$ btx using the same vector and otherwise identical assay conditions (Hruska et al., 2007). The mean response of the PSCA-expressing neurons cannot be significantly lowered by the addition of a solution containing the $\alpha 7$-nAChRspecific antagonist, $\alpha$ btx (Fig. $5 B$ ). Inclusion of $\alpha$ btx to openRCASBP(A) infected neuronal cultures reduces the amplitude of $\mathrm{Ca}^{2+}$ transients to the levels that are comparable to the PSCA infected neurons (Fig. $5 B ; p<0.001$, one-way ANOVA, Bonferroni post test: open: $0.3 \pm 0.02, n=69$, open + btx: $0.14 \pm 0.02$, $n=31$ ). Thus, retrovirally delivered PSCA expressed at E8 in ciliary ganglion neurons appears to primarily act to suppress activation of $\alpha 7$-nAChRs, but this suppression is less than that observed for tethered $\alpha$ btx, suggesting that the PSCA affects a 
A
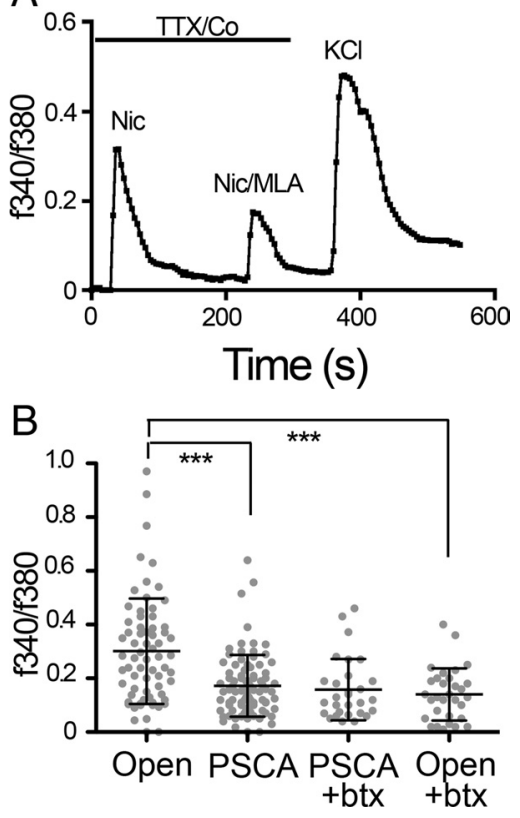

Figure 5. Overexpression of PSCA at E8 blocks nicotine-induced calcium influx via $\alpha 7$ $n A C h R s$. Ciliary ganglia from embryos infected with RCASBP(A)-PSCA or RCASBP(A) open (without an insert) at St. 8-9 were isolated at E8, dissociated, and loaded with Fura-2, $2 \mathrm{~h}$ after plating. Nicotine $(10 \mu \mathrm{M})$ was used to stimulate nAChRs in the presence TTX and cobalt, which block voltage activated influx of calcium. $\boldsymbol{A}$, Brief application of nicotine induces rapid increase in $\left[\mathrm{Ca}^{2+}\right]_{\mathrm{i}}$. The $\alpha 7$-specific antagonist MLA blocks $50 \%$ of this nicotine-induced response. The third response is the total calcium signal observed in response to perfusion with $25 \mathrm{~mm} \mathrm{KCl}$ in the absence of TTX and $\mathrm{C}^{2+}$. The mean peak $\mathrm{Ca}^{2+}$ responses due to depolarization with $25 \mathrm{~mm} \mathrm{KCl}$ between open and PSCA infected ciliary ganglion neurons are not significantly different, indicating the all neurons are equally loaded with Fura-2. $\boldsymbol{B}$, Plot of all the peak nicotine-induced calcium responses observed in PSCA versus open virus infected neurons. Each gray spot represents the response of a single neuron. Graph represents the sum of data from 3 independent experiments using viral injections together with calcium imaging of E8 neurons. PSCA infected neurons exhibit a significantly smaller mean nicotine-induced increase in $\left[\mathrm{Ca}^{2+}\right]_{i}$ compared with open-infected neurons. Bars indicate mean plus or minus SD ( $p<0.001$; open: $0.3 \pm$ $0.02, n=69 ;$ PSCA: $0.17 \pm 0.01, n=86$ ). Addition of exogenous $\alpha$ btx ( $50 \mathrm{~nm}$ ) to PSCA infected neurons does not cause additional reduction in the mean nicotine-induced $\mathrm{Ca}^{2+}$ whereas addition of $\alpha$ btx on open infected neurons significantly reduces increases in $\left[\mathrm{Ca}^{2+}\right]_{\mathrm{i}}(p<$ 0.001; open: $0.3 \pm 0.02, n=69$; open +btx: $0.14 \pm 0.02 n=31$ ), whereas open with PSCA is not significantly different from open +btx or PSCA +btx; one-way ANOVA with Bonferroni post hoc test.

subpopulation of neurons or a subpopulation of the nicotinic responses.

Since signaling through $\alpha 7$-nAChRs affects the final number of neurons in the ciliary ganglion, we determined whether the premature expression of PSCA caused a change in neuronal survival. We quantified the total number of neurons (using anti-Islet-1) as well as the number of choroid neurons (using anti-somatostatin) at E14 by using design-based stereology (Lee et al., 2001; Bunker and Nishi, 2002). If infective RCASBP(A)PSCA particles are injected at St. 8-9 (36 h of incubation), virtually all of the ciliary ganglion neurons are infected (Fig. 6A-C); however, if embryos are injected at St. 10-13 (48 h of development), very few neurons are infected, whereas many surrounding non-neuronal cells are infected (Fig. 6D-F). Little or no infection is detected in the accessory oculomotor nucleus, which innervates the ciliary ganglion (Fig. 6G-I). PSCA expressing ganglia infected at $36 \mathrm{~h}$ of development contained 35\% more choroid neurons than ganglia infected with open-RCASBP(A) (Fig. 7; $p<$ 0.0001, one-way ANOVA; open: $4679 \pm 353.4, n=16$; PSCA at
36 h: $7125 \pm 355.1, n=13$ ) and this was reflected in a significant change in the total number of neurons in the ganglion $(p<0.001$ one-way ANOVA; open: $8626 \pm 418.8, n=16$; PSCA at $36 \mathrm{~h}$ : $11,173 \pm 467, n=13)$. This indicates that the change in the number of neurons expressing somatostatin-like immunoreactivity was not merely due to a shift in neuropeptide expression. Interestingly, the number of ciliary neurons remained unchanged (Fig. 7), suggesting that choroid neurons are more sensitive to the expression of PSCA. When RCASBP(A)-PSCA retrovirus is injected into the neural tube at St. 10-13, then PSCA fails to rescue neurons from dying (Fig. 7; PSCA at 48 h: $9352 \pm 646.1, n=9$ ), despite the fact that neighboring glia are infected and overexpress PSCA.

\section{Discussion}

The principal finding of this study is that PSCA, a molecule originally identified as an antigen upregulated in prostate cancer, is an endogenous prototoxin highly expressed in the nervous system that attenuates signaling through $\alpha 7$ subunit-containing nAChRs. PSCA levels correlate with the expression of transcripts encoding $\alpha 7$-nAChRs. In the avian ciliary ganglion, PSCA expression is induced and upregulated between E8 and E14, a time during which half of the neurons are lost by cell death and peripheral synaptogenesis is completed. Furthermore, forcing premature expression of PSCA blocks $\alpha 7 \mathrm{nAChR}$ activation and prevents choroid neurons from dying. These studies uncover possible modulatory functions for prototoxins during development.

PSCA belongs to the Ly6 superfamily, whose members possess cysteine rich molecules expressed in tissue-specific patterns during development and in the adult (Gumley et al., 1995). To date, the function of such molecules has been mysterious because their small size and lack of a transmembrane domain preclude a direct role in mediating cell signaling. Within this family, molecules that fold into a structure homologous to those formed by cobratoxins (Tsetlin, 1999), which bind with high affinity to specific subclasses of nAChRs, have been described as prototoxins and include mouse lynx1 (Miwa et al., 1999), mouse lynx2 (Dessaud et al., 2006), Ly6H (Horie et al., 1998) and SLURP-1 (Chimienti et al., 2003). These endogenous prototoxins are expressed in the CNS and the PNS, and likely act as molecules that interact cell autonomously to modulate nicotinic receptor function in vivo. Indeed, lynx1 and lynx 2 coprecipitate with $\alpha 4 / \beta 2$ as well as $\alpha 7$ subunit-containing nAChRs in mouse CNS and this association alters nAChR kinetics, extent of receptor desensitization and agonist affinity (Miwa et al., 1999; Ibañez-Tallon et al., 2002; Tekinay et al., 2009). Furthermore, neurons from lynx1-null mice exhibit large increases in $\left[\mathrm{Ca}^{2+}\right]_{\mathrm{i}}$ in response to nicotine and, as a result, display age-dependent degeneration that is exacerbated by nicotine and ameliorated by null mutations in nAChRs (Miwa et al., 2006), and lynx2-null mice have altered responses to fear conditioning (Tekinay et al., 2009). Our studies using retroviralmediated expression of avian PSCA in chicken neurons indicate that PSCA exhibits many of the features of a prototoxin: (1) it is cysteine-rich, with a spacing of cysteine residues that is conserved with other members of the family; (2) it is highly expressed in tissues of the nervous system that contain high levels of $\alpha 7$ $n A C h R s$ and these data are corroborated by in situ hybridization from mouse brain, where PSCA is detected in Purkinje and granule cell layers of cerebellum, cerebral cortex and hippocampus (http://www.brain-map.org/mouse/brain/psca.html); (3) it is predicted to form a "three-fingered" tertiary structure similar to that of nicotinic antagonists derived from cobratoxin; and (4) it interferes with nicotine-induced increases in $\left[\mathrm{Ca}^{2+}\right]_{i}$ through 


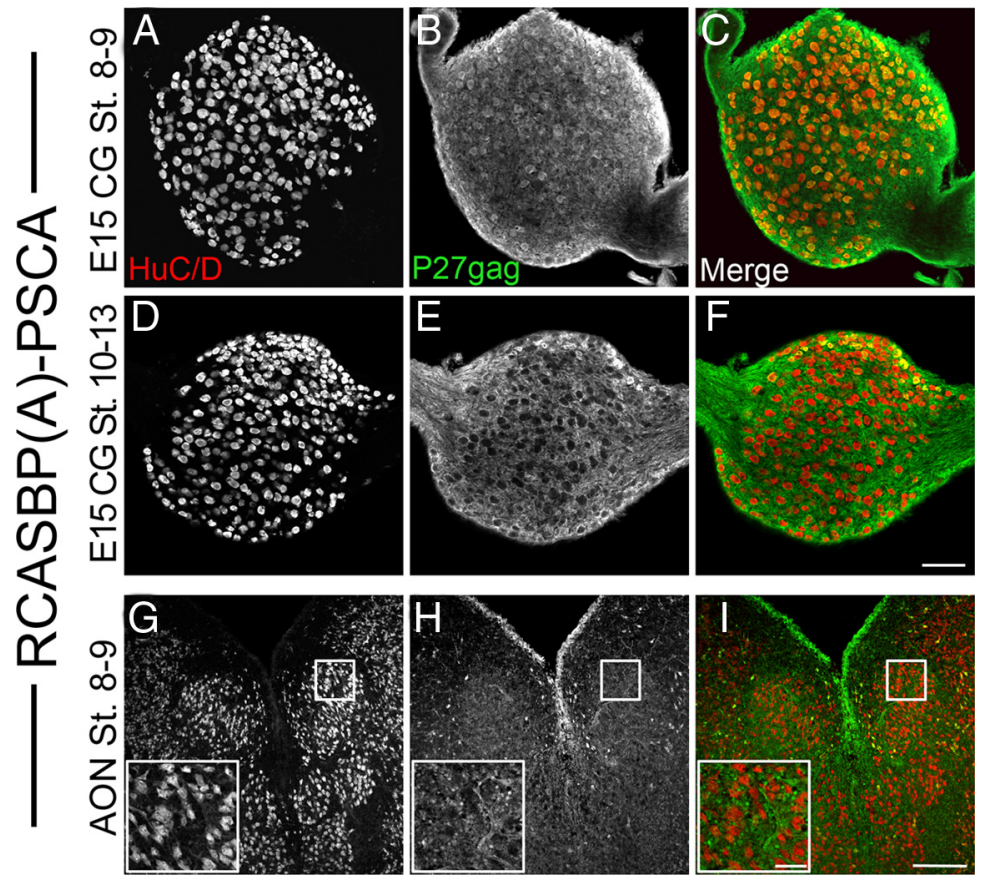

Figure 6. Timing of viral injection directs PSCA gene expression to ciliary ganglion neurons but not preganglionic oculomotor neurons. Embryos were injected with RCASBP(A)-PSCA at $36 \mathrm{~h}$ of development (St. 8-9; $A-C$ ) and 48 h of development (St. $10-13$; $\boldsymbol{D}-\boldsymbol{F}) \cdot \boldsymbol{A}-\boldsymbol{C}, \mathrm{p} 27$ gag immunoreactivity (green) is observed in $>80 \%$ of neurons (red) in ciliary ganglia from embryos infected at St. 8-9.D-F, Only surrounding non-neural tissue exhibits p27gag immunoreactivity in the ciliary ganglia from embryos infected at St. $10-13$. Calibration bar $=100 \mu \mathrm{m} . \mathbf{G}, \boldsymbol{H}$, Neurons in the preganglionic AON from the embryos injected at St. $8-9$ are not infected and only surrounding glia exhibits p27gag immunoreactivity. Calibration bar $=250 \mu \mathrm{m}$ (inset $=50 \mu \mathrm{m}$ ).

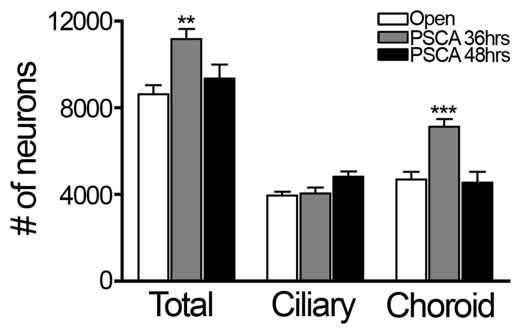

Figure 7. Premature expression of chPSCA at E8 rescues choroid neurons from dying. Serially sectioned E14 ciliary ganglia infected with RCASBP(A)-PSCA at St. 8-9 (36 h of incubation) or St. 10-13 (48 h of incubation) were labeled with Islet-1 antibody to stain all the neurons and somatostatin antibody to label choroid neurons. Number of surviving neurons was determined using designbased stereology. Ciliary ganglia infected at St. 8-9 have significantly more choroid neurons ( $p<0.001$; open: $4679 \pm 353.4, n=16$; PSCA at $36 \mathrm{~h}: 7125 \pm 355.1, n=13)$ compared with the open-infected ganglia. The number of ciliary neurons is the same in open and RCASBP(A)-PSCA infected ciliary ganglia. The total number of neurons is also significantly greater in RCASPB(A)-PSCA ganglia infected at St. $8-9(p<0.002)$. Infection at St. $10-13$ does not prevent cell death of ciliary or choroid neurons. Values represent mean \pm SEM of three or more separate experiments. ANOVA with Tukey's multiple-comparison post hoc test was used to analyze the results.

$\alpha 7$-nAChRs, but not heteromeric nAChRs. These results suggest that many other members of the Ly6 superfamily may also serve as prototoxins with selectivity for specific classes of nAChRs.

Nicotinic signaling has long been known to play an important role in regulating programmed cell death during development. Blocking neuromuscular transmission with nicotinic antagonists such as D-tubocurarine or $\alpha$-bungarotoxin is one of the most effective ways to rescue spinal cord motor neurons from dying (Pittman and Oppenheim, 1978, 1979). In the autonomic and some parts of the CNS, activation of neuronal nAChRs can directly induce apoptosis. For example, chronic blockade of $\alpha 7$ nAChRs with systemically applied $\alpha$ btx or MLA prevents cell death of nearly all ciliary ganglion neurons (Meriney et al., 1987; Bunker and Nishi, 2002), although the same is not true for spinal cord motor neurons (Oppenheim et al., 2000). In addition, reduction in $\mathrm{Ca}^{2+}$ influx through $\alpha 7$-nAChRs in a cellautonomous manner prevents ciliary and choroid neurons from dying, suggesting that large $\alpha 7$-nAChR-mediated increases in $\left[\mathrm{Ca}^{2+}\right]_{i}$ promote cell death of ciliary ganglion neurons during development (Hruska et al., 2007). Immature neurons are especially vulnerable to $\mathrm{Ca}^{2+}$ influx via $\alpha 7$-nAChRs; activation of these channels induces apoptosis of hippocampal progenitor cells but not differentiated hippocampal neurons (Berger et al., 1998). Since the activation of $\alpha 7$-nAChRs can be proapoptotic in certain neuronal populations, the signaling through these channels must be precisely regulated.

Our data are consistent with PSCA acting as a modulator of nicotinic signaling in the ciliary ganglion that limits cell death caused by activation of $\alpha 7-n A C h R s$ in neurons vulnerable to calcium overload. At E8, when PSCA is undetectable, many ciliary ganglion neurons are undergoing apoptotic cell death (Lee, 2001). Subsequent upregulation of PSCA correlates with a significant decrease in cell death, reflected in the stabilization of neuronal cell number. Overexpression of PSCA at E8, when it is not normally expressed, blocks nicotine-induced increases in intracellular calcium through $\alpha 7$ nAChRs and prevents cell death of choroid but not ciliary neurons. Finally, blocking all $\alpha 7$ signaling with $\alpha$ btx attenuates the developmental increase seen in PSCA, consistent with calcium influx upregulating PSCA as part of a negative feedback loop.

The selective effect of PSCA on the choroid neuron subpopulation contrasts to that of membrane-tethered $\alpha$ btx, which also blocks nicotine-induced increases in $\left[\mathrm{Ca}^{2+}\right]_{\mathrm{i}}$ via $\alpha 7$-nAChRs (Hruska et al., 2007), but rescues both ciliary and choroid subpopulations. One possible explanation is that tethered $\alpha \mathrm{btx}$ blocks a much higher percentage of the mean nicotine-induced response when compared with PSCA (69\% versus 43\%) (Hruska et al., 2007). One likely difference between $\alpha$ btx and PSCA may be the dissociation constants when bound to $\alpha 7$-nAChRs. The dissociation constant of $\alpha$ btx is so high that it is virtually irreversible, whereas this is unlikely to be the case for PSCA. Thus, PSCA may have a differential affinity for choroid neuron nAChRs, or ciliary neuron nAChRs may already be occupied by a prototoxin that does not block calcium influx but cannot be displaced by PSCA. This may be likely because we find two other prototoxinlike molecules, ch3Ly and ch5Ly, in the ciliary ganglion. This differential susceptibility of choroid neurons to PSCA warrants further investigation, but is difficult to pursue because extracellular markers that distinguish ciliary from choroid neurons in acutely dissociated, live preparations have yet to be discovered. Thus, ciliary versus choroid neurons cannot be definitively identified for electrophysiological studies and neither can they be conveniently sorted for biochemical binding or molecular studies. In addition, the measurement of the binding and dissociation 
constants of membrane-tethered molecules to integral membrane proteins is highly challenging.

The relationship of PSCA expressed in the prostate to its expression in the nervous system is not clear. PSCA was first identified as an antigen enriched in basal cells of the prostate epithelium that is upregulated in high grade, metastatic prostate tumors (Reiter et al., 1998), but undetectable in northern blots of whole human brain. In contrast, when using the more sensitive quantitative PCR on dissected neural tissues, we see significantly higher levels of PSCA than in peripheral organs. Currently, it is unknown whether PSCA modulates $\alpha 7$-nAChR signaling in the prostate or whether it has a completely unrelated function. However, many non-neural cells, such as keratinocytes, lymphocytes, endothelial cells and glia, express $\alpha 7$-nAChRs (for review, see Sharma and Vijayaraghavan, 2002). In this context, SLURP1 and 2 of the Ly6, lynx superfamily, regulate apoptosis in keratinocytes (Chimienti et al., 2003; Arredondo et al., 2006, 2007). Thus, It is possible that $\alpha 7$-nAChR signaling also regulates cell proliferation in the prostate. Therefore, uncovering the neural and non-neural functions of $\mathrm{nAChRs}$ and their accompanying prototoxin modulators will be key for understanding the importance of nicotinic signaling in normal physiology and disease.

\section{References}

Arredondo J, Chernyavsky AI, Jolkovsky DL, Webber RJ, Grando SA (2006) SLURP-2: a novel cholinergic signaling peptide in human mucocutaneous epithelium. J Cell Physiol 208:238-245.

Arredondo J, Chernyavsky AI, Grando SA (2007) SLURP-1 and -2 in normal, immortalized and malignant oral keratinocytes. Life Sci 80:2243-2247.

Berger F, Gage FH, Vijayaraghavan S (1998) Nicotinic receptor-induced apoptotic cell death of hippocampal progenitor cells. J Neurosci 18:6871-6881.

Blumenthal EM, Shoop RD, Berg DK (1999) Developmental changes in the nicotinic responses of ciliary ganglion neurons. J Neurophysiol 81:111-120.

Bunker GL, Nishi R (2002) Developmental cell death in vivo: rescue of neurons independently of changes at target tissues. J Comp Neurol 452:80-92.

Chimienti F, Hogg RC, Plantard L, Lehmann C, Brakch N, Fischer J, Huber M, Bertrand D, Hohl D (2003) Identification of SLURP-1 as an epidermal neuromodulator explains the clinical phenotype of Mal de Meleda. Hum Mol Genet 12:3017-3024.

Conroy WG, Berg DK (1995) Neurons can maintain multiple classes of nicotinic acetylcholine receptors distinguished by different subunit compositions. J Biol Chem 270:4424-4431.

Darland DC, Link BA, Nishi R (1995) Activin A and follistatin expression in developing targets of ciliary ganglion neurons suggests a role in regulating neurotransmitter phenotype. Neuron 15:857-866.

Dessaud E, Salaün D, Gayet O, Chabbert M, deLapeyrière O (2006) Identification of lynx2, a novel member of the ly-6/neurotoxin superfamily, expressed in neuronal subpopulations during mouse development. Mol Cell Neurosci 31:232-242.

Federspiel MJ, Hughes SH (1997) Retroviral gene delivery. Methods Cell Biol 52:179-214.

Gumley TP, McKenzie IF, Sandrin MS (1995) Tissue expression, structure and function of the murine Ly- 6 family of molecules. Immunol Cell Biol 73:277-296.

Horie M, Okutomi K, Taniguchi Y, Ohbuchi Y, Suzuki M, Takahashi E (1998) Isolation and characterization of a new member of the human Ly6 gene family (LY6H). Genomics 53:365-368.

Hruska M, Ibañez-Tallon I, Nishi R (2007) Cell-autonomous inhibition of alpha 7-containing nicotinic acetylcholine receptors prevents death of parasympathetic neurons during development. J Neurosci 27:11501-11509.

Ibañez-Tallon I, Miwa JM, Wang HL, Adams NC, Crabtree GW, Sine SM, Heintz N (2002) Novel modulation of neuronal nicotinic acetylcholine receptors by association with the endogenous prototoxin lynx1. Neuron 33:893-903.

Landmesser L, Pilar G (1972) The onset and development of transmission in the chick ciliary ganglion. J Physiol 222:691-713.

Landmesser L, Pilar G (1974) Synaptic transmission and cell death during normal ganglionic development. J Physiol 241:737-749.

Lee VM (2001) Regulation of neuronal cell number in the developing ciliary ganglion. In: Cell and developmental biology, p 137. Portland: Oregon Health Sciences University.

Lee VM, Smiley GG, Nishi R (2001) Cell death and neuronal replacement during formation of the avian ciliary ganglion. Dev Biol 233:437-448.

Maderdrut JL, Oppenheim RW, Prevette D (1988) Enhancement of naturally occurring cell death in the sympathetic and parasympathetic ganglia of the chicken embryo following blockade of ganglionic transmission. Brain Res 444:189-194.

Marusich MF, Weston JA (1992) Identification of early neurogenic cells in the neural crest lineage. Dev Biol 149:295-306.

McNerney ME, Pardi D, Pugh PC, Nai Q, Margiotta JF (2000) Expression and channel properties of alpha-bungarotoxin-sensitive acetylcholine receptors on chick ciliary and choroid neurons. J Neurophysiol 84:1314-1329.

Meriney SD, Pilar G, Ogawa M, Nunez R (1987) Differential neuronal survival in the avian ciliary ganglion after chronic acetylcholine receptor blockade. J Neurosci 7:3840-3849.

Miwa JM, Ibanez-Tallon I, Crabtree GW, Sánchez R, Sali A, Role LW, Heintz N (1999) lynx1, an endogenous toxin-like modulator of nicotinic acetylcholine receptors in the mammalian CNS. Neuron 23:105-114.

Miwa JM, Stevens TR, King SL, Caldarone BJ, Ibanez-Tallon I, Xiao C, Fitzsimonds RM, Pavlides C, Lester HA, Picciotto MR, Heintz N (2006) The prototoxin lynx1 acts on nicotinic acetylcholine receptors to balance neuronal activity and survival in vivo. Neuron 51:587-600.

Morgan BA, Fekete DM (1996) Manipulating gene expression with replication-competent retroviruses. Methods Cell Biol 51:185-218.

Oppenheim RW, Prevette D, D'Costa A, Wang S, Houenou LJ, McIntosh JM (2000) Reduction of neuromuscular activity is required for the rescue of motoneurons from naturally occurring cell death by nicotinic-blocking agents. J Neurosci 20:6117-6124.

Pittman R, Oppenheim RW (1979) Cell death of motoneurons in the chick embryo spinal cord. IV. Evidence that a functional neuromuscular interaction is involved in the regulation of naturally occurring cell death and the stabilization of synapses. J Comp Neurol 187:425-446.

Pittman RH, Oppenheim RW (1978) Neuromuscular blockade increases motoneurone survival during normal cell death in the chick embryo. Nature 271:364-366.

Pugh PC, Margiotta JF (2000) Nicotinic acetylcholine receptor agonists promote survival and reduce apoptosis of chick ciliary ganglion neurons. Mol Cell Neurosci 15:113-122.

Reiter RE, Gu Z, Watabe T, Thomas G, Szigeti K, Davis E, Wahl M, Nisitani S, Yamashiro J, Le Beau MM, Loda M, Witte ON (1998) Prostate stem cell antigen: a cell surface marker overexpressed in prostate cancer. Proc Natl Acad Sci U S A 95:1735-1740.

Sharma G, Vijayaraghavan S (2002) Nicotinic receptor signaling in nonexcitable cells. J Neurobiol 53:524-534.

Tekinay AB, Nong Y, Miwa JM, Lieberam I, Ibanez-Tallon I, Greengard P, Heintz N (2009) A role for LYNX2 in anxiety-related behavior. Proc Natl Acad Sci U S A 106:4477-4482.

Tsetlin V (1999) Snake venom alpha-neurotoxins and other 'three-finger' proteins. Eur J Biochem 264:281-286.

Tsuji H, Okamoto K, Matsuzaka Y, Iizuka H, Tamiya G, Inoko H (2003) SLURP-2, a novel member of the human Ly-6 superfamily that is upregulated in psoriasis vulgaris. Genomics 81:26-33.

Wang L, Galehouse D, Mellon P, Duesberg P, Mason WS, Vogt PK (1976) Mapping oligonucleotides of Rous sarcoma virus RNA that segregate with polymerase and group-specific antigen markers in recombinants. Proc Natl Acad Sci U S A 73:3952-3956.

Wright L (1981) Cell survival in chick embryo ciliary ganglion is reduced by chronic ganglionic blockade. Brain Res 227:283-286. 